\title{
Atrial fibrillation influences automatic oscillometric ankle-brachial index measurement
}

\author{
Michał Dąbrowski, Jacek Lewandowski, Cezary Szmigielski, Maciej Siński
}

Department of Internal Medicine, Hypertension and Vascular Diseases, Medical University of Warsaw, Warsaw, Poland

Submitted: 17 January 2018; Accepted: 22 March 2018; Online publication: 12 July 2019

Arch Med Sci 2021; 17 (3): 621-627

DOI: https://doi.org/10.5114/aoms.2018.75891

Copyright $\odot 2019$ Termedia \& Banach

\section{Abstract}

Introduction: Repeated measurements of ankle-brachial index (ABI) using Doppler method were shown to be accurate during atrial fibrillation. Oscillometric devices are effective in $A B I$ measurement, but their accuracy during atrial fibrillation is unknown. The purpose of the study was to investigate whether atrial fibrillation influences $A B I$ obtained with the automatic oscillometric method.

Material and methods: Ninety-nine patients with atrial fibrillation (mean age: $66.6+(S D=11)$ years, $M / F-63 / 36)$ who underwent electrical cardioversion were investigated (198 lower extremities). The ABI measurements using oscillometric and Doppler methods were performed on both lower extremities before and after procedure.

Results: The $A B I$ measured using the oscillometric method on both lower limbs did not change after cardioversion (1.21 (IQR: 1.13-1.27) vs. 1.22 (IQR: $1.14-1.26), p=0.664$, respectively). The $A B I$ measured before and after cardioversion using Doppler and oscillometric methods showed a significant difference (1.14 (IQR: $1.07-1.22$ ) vs. 1.21 (IQR: $1.13-1.27), p<0.001$ and 1.18 (IQR: $1.09-1.13$ ) vs. 1.22 (IQR: $1.14-1.26), p<0.001$ respectively). Both methods showed a weak correlation before $(r=0.35, p<0.001)$ and no correlation after cardioversion $(r=0.12, p=0.07)$. The Bland-Altman plot showed poor agreement between measurements performed with the Doppler and oscillometric methods in sinus rhythm and during atrial fibrillation. Conclusions: The automated oscillometric method of $A B I$ measurements should not replace the reference Doppler method in patients with atrial fibrillation. More research related to the oscillometric measurements is needed in subjects with peripheral artery disease and atrial fibrillation.

Key words: ankle-brachial index, atrial fibrillation, oscillometric method.

\section{Introduction}

Peripheral artery disease (PAD) is associated with significant morbidity, mortality and quality of life impairment [1-3]. The ankle-brachial index $(A B I)$ is a noninvasive, safe, simple and quick to perform test for lower extremity $\operatorname{PAD}[2,4]$. The critical evidence gaps and future research directions for PAD-related research include advancement in diagnostics, and technologies for highly accurate measurement of $A B I$ [1].

The $A B I$ is the ratio of the systolic blood pressure (SBP) measured at the ankle to that measured at the brachial artery $[5,6]$. In a clinical setting, many factors influence the $A B I$ measurements, including method of measurement, repeated measures, as well as demographic parameters

\author{
Corresponding author: \\ Dr. Maciej Siński \\ Department of Internal \\ Medicine, Hypertension \\ and Vascular Diseases \\ Medical University of Warsaw \\ Banacha 1a \\ 02-097 Warsaw, Poland \\ Phone +48 225991828 \\ Fax +48 225992828 \\ E-mail: msinski@wum.edu.pl
}


including height, gender, race or heart rate [7]. Especially in patients with atrial fibrillation (AF), the $A B I$ may be potentially more difficult because of high beat-to-beat variability of heart rate and stroke volume [8]. Our previous study showed that $A F$ does not significantly affect $A B I$ measured by the Doppler method [9]. However, there is still a lack of data indicating whether the automatic oscillometric method, often used for $A B I$ measurements, is also accurate in AF patients. A meta-analysis of 24 studies assessing the usefulness of automated oscillometric devices for $\mathrm{ABI}$ estimation, when compared with the Doppler method, showed that the oscillometric method may be a useful alternative to the Doppler method in patients without atrial fibrillation [10]. Atrial fibrillation does not significantly affect the accuracy of oscillometric blood pressure measurements when three repeated measurements are performed [11]. However, these findings were questioned because of specific validation criteria [12]. Hence, the answer as to how AF affects the oscillometric blood pressure and $A B I$ measurements remains not fully known.

Therefore, the purpose of this study was to investigate whether atrial fibrillation influences results of $A B I$ measurements performed with an automatic oscillometric device.

\section{Material and methods}

\section{Study design}

In this study, we included data from one hundred and fifteen consecutive patients with atrial fibrillation admitted for electrical cardioversion (EC). The current study is the extension of the research published by Dabrowski et al. [9], where detailed patients' characteristics were presented. Ninety-nine patients (mean age: $66.6 \pm 11$ ) years, M/F - 63/36) underwent the complete study protocol and were analyzed (Figure 1).

The inclusion criteria for participation in the study were age 18-70 years and clinical decision of patients' eligibility for electrical cardioversion (EC) of atrial fibrillation. The exclusion criteria were circulatory instability, use of vasoconstrictive agents, limb trauma or upper limb artery stenosis.

The local bioethical committee accepted the study and informed consent was obtained from all participants. This trial is registered at ClinicalTrials.gov (NCT02986282).

$A B I$ was performed in two study sessions, before and after EC. The first session was scheduled before routine anesthesia for EC (with intravenous propofol 1\%). The second session started approximately $1 \mathrm{~h}$ after EC, in the same intensive care unit, with the patient fully conscious. All study-related procedures were finished within $2 \mathrm{~h}$ after EC. No adverse events were observed during the study-related procedures in the intensive care unit.

\section{$A B I$ measurements}

All the measurements were taken in the intensive care unit at an ambient room temperature of about $21^{\circ} \mathrm{C}$, after patients gave their written informed consent to participate in the study. All

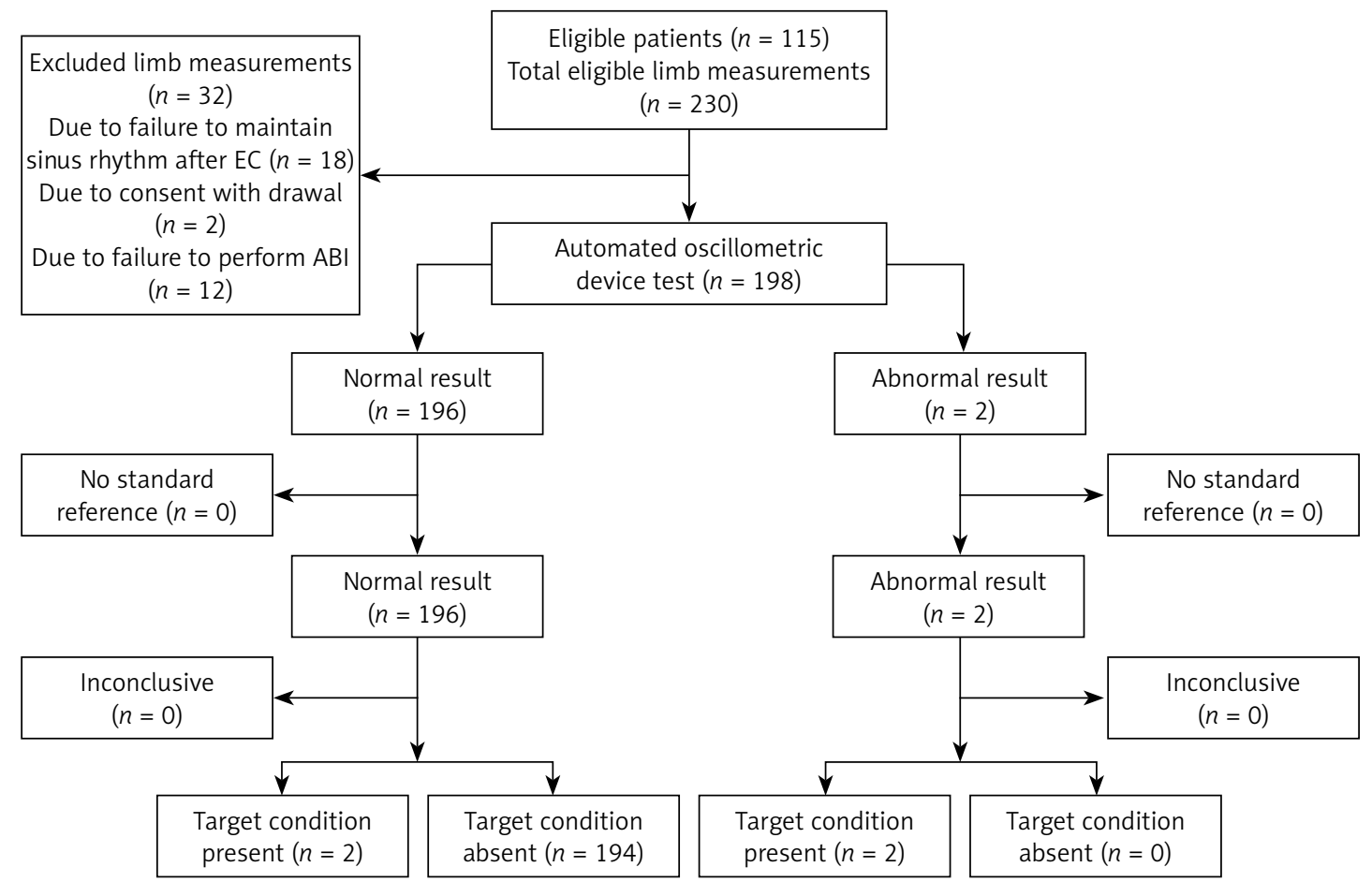

Figure 1. STARD (Standards for Reporting of Diagnostic Accuracy) flowchart representing the selection of the study patients 
patients were awake, fasting and in the supine position. The ankle-brachial index was measured according to the guidelines issued by AHA [7]. Firstly, Doppler ABI measurements were performed. Systolic blood pressure (SBP) was measured using a Doppler device (Echo Sounder ES-101EX, Hadeco, Kawasaki, Japan) and a validated and calibrated aneroid sphygmomanometer (Minimus II, Riester, Jungingen, Germany). For each measurement, the appropriate cuff size was used, with the cuff width measuring at least $40 \%$ of the limb circumference. The higher value of SBP measured on the arm and the higher value of SBP measured on the posterior tibial or dorsalis pedis artery were used to calculate the ABI. During both study sessions throughout the study, the $A B I$ measurements were repeated 3 times in an alternating clockwise sequence. The clockwise sequence was as follows: right arm, right posterior tibial, right dorsalis pedis, left posterior tibial, left dorsalis pedis, left arm, right arm. The counterclockwise sequence started with the left arm, with each successive set of measurements taken in the reverse order. The same sequence of limb pressure measurements was used during the whole study. The measurements were repeated three times, and the mean of the results was used for calculations. After Doppler measurements, the WatchBP Office ABI system (MicroLife WatchBP AG, Windau, Switzerland) was used for three measurements on both lower extremities. The mean of three $A B I$ measurements was used for further calculations. The $A B I$ system included two sizes of arm cuffs and ankle cuff. All automated oscillometric recordings and Doppler measurements were performed using appropriately sized cuffs. One investigator (M.D.), trained in vascular procedures, performed all $A B I$ measurements.

\section{Endpoints}

The primary endpoint of this study was the difference between $A B I$ measures before and after electrical cardioversion using the oscillometric method, and additionally the difference between measurements performed with the Doppler method and the oscillometric method.

\section{Statistical analysis}

A sample size calculation was made for the initial study and presented in our previous publication [9]. Each lower extremity measurement was considered as a separate test; therefore, 198 measurements were repeated 3 times using both the Doppler and the oscillometric method. The data were processed with the Shapiro-Wilk normality test. The $A B I$ measurements were compared using the Wilcoxon signed rank test. The comparison was calculated for patients with sinus rhythm and during atrial fibrillation, as well as for measures with the two studied methods. For normal data distributions, the paired two-tailed $t$ test was used. The Bland-Altman plot was used to analyze the agreement between $A B I$ measurements performed in sinus rhythm with an oscillometric device (considered the method tested in standard conditions) and $\mathrm{ABI}$ measurements performed during atrial fibrillation (method tested in experimental conditions). The Bland and Altman plot was also used to analyze the agreement between the $A B I$ measured by the reference Doppler method and the $A B I$ measured by the oscillometric device in experimental conditions. Correlations between $A B I$ measurements in sinus rhythm and after $E C$ using the oscillometric method were performed. Also, the correlation between both methods of $\mathrm{ABI}$ and the Spearman's rho coefficient were estimated. Statistical significance was established at $p<0.05$. All data were calculated using statistical software R 3.1.3 and were expressed as median $(\mathrm{IQR})$ and mean (SD) as appropriate.

\section{Results}

Using the reference Doppler method we investigated in total 198 lower extremities in 99 patients referred for EC. The diagnosis of PAD, as defined by $A B I \leq 0.9$, was established in 3 patients $(A B I \leq 0.9$ was detected on 4 lower extremities). The automated oscillometric method detected 2 patients with PAD (two lower extremities with $A B I \leq 0.9$; Figure 1) in sinus rhythm and 1 during atrial fibrillation (one lower extremity). Eight borderline PAD ( $A B I$ in the range of 0.91-1.0) were identified by the Doppler method and 10 using the oscillometric method.

The median $A B I$ measurement with the oscillometric method, performed on both lower limbs before $E C$, did not differ from $A B I$ after restoration to sinus rhythm (1.21 (1.13-1.27) before EC vs. 1.22 (1.14-1.26) after EC, $p=0.664$, respectively) (Figure 2). A correlation was found between measurements performed with the oscillometric method before and after EC on both lower limbs $(r=0.49, p<0.001)$ (Figure 3). The Bland-Altman plot was performed, using the measurement after EC as the reference. The average bias was low 0.009 (95\% limits of agreement -0.22 and 0.24 ); however, more than $5 \%$ of differences were beyond the limits of agreement, suggesting poor agreement between measurements performed with the oscillometric method before and after cardioversion (Figure 3).

When compared with the Doppler measurements, the oscillometric measurements were significantly higher both during AF (1.21 (1.13-1.27) vs. 1.14 (1.07-1.22), $p<0.001$ respectively) and in sinus rhythm (1.22 (1.14-1.26) vs. 1.18 


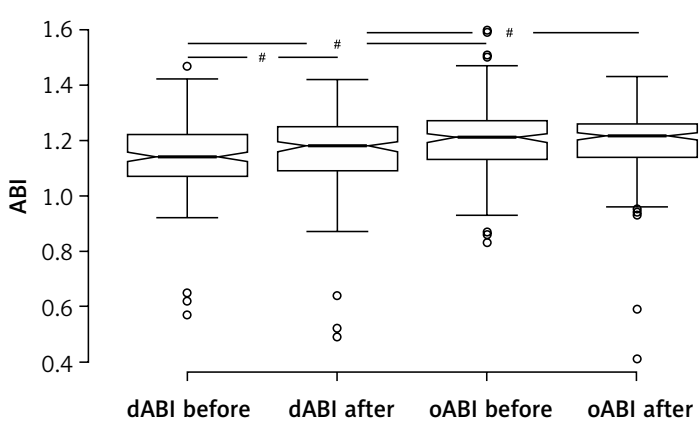

Figure 2. Plot showing differences in median ankle-brachial index $(A B I)$ before and after EC assessed using Doppler and oscillometric method respectively - $\mathrm{dABI}$ before, $\mathrm{dABI}$ after, oABI before and $\mathrm{OABI}$ after, $\# p<0.001$. The dots indicate outliers that are more than $3 / 2$ of the upper quartile, and the whiskers indicate maximum and minimum excluding outliers, the box margins show upper $(75 \%)$ and lower quartile (25\%), and the central line represents the median

(1.09-1.13), $p<0.001$ respectively; Figure 2). Upper $95 \% \mathrm{Cl}$ margins for the median of difference in $A B I$ between methods were 0.08 and 0.06 for the results obtained before and after cardioversion. The difference was higher during AF than during sinus rhythm. That may be significant from a clinical perspective (0.07 vs. $0.04, p=0.002$ ).

The two methods showed a weak correlation before EC $(r=0.35, p<0.001)$, but after EC there was no correlation between the methods $(r=0.12$, $p=0.07$; Figure 4). The Bland-Altman plot showed no significant agreement between measurements performed using the oscillometric method and the Doppler method in sinus rhythm and during AF (Figure 5). The average bias before EC was 0.068 (95\% limits of agreement -0.21 and 0.35 ) and after EC was 0.035 (95\% limits of agreement -0.30 and 0.37).

\section{Discussion}

Guidelines for PAD recommends the $A B I$ as a strong, class 1 recommendation, of moderate-quality evidence as a diagnostic test in patients with history or physical examination findings suggestive of PAD $[1,2]$. This test is also important for follow-up and periodic evaluation in patients with PAD who have undergone lower extremity revascularization. A change in $A B I$ of 0.15 is considered clinically significant $[1,13]$.

A

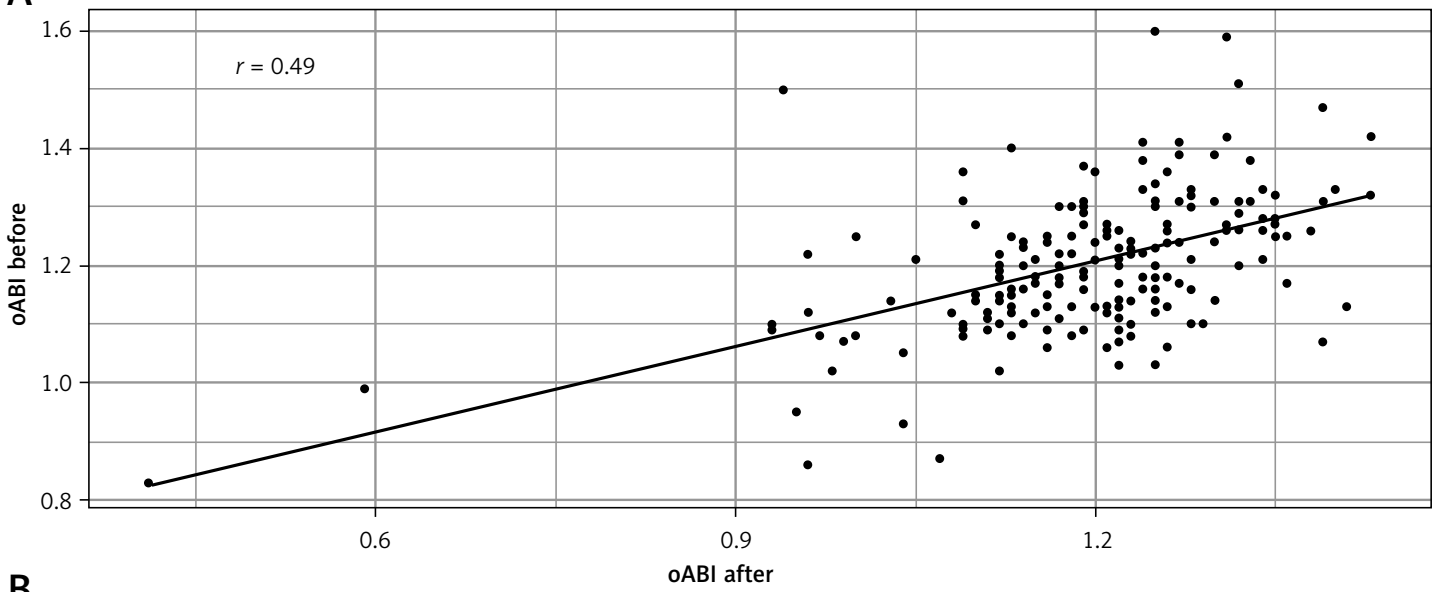

B

oABI after

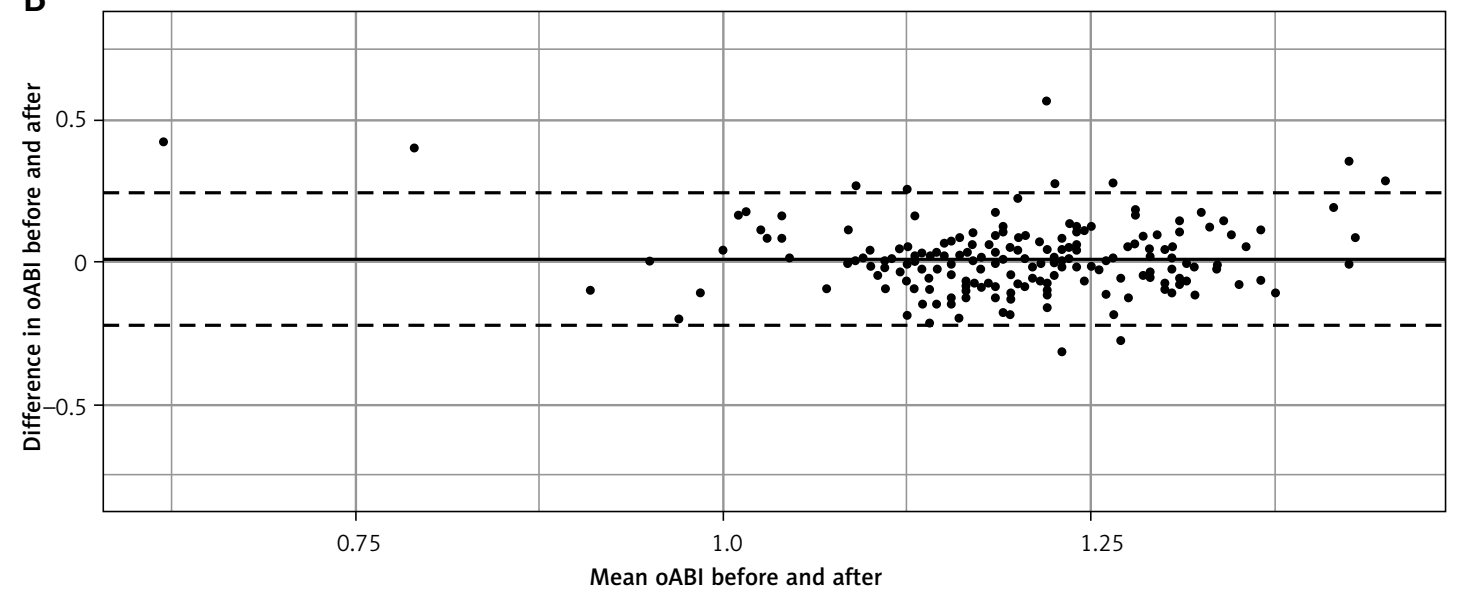

Figure 3. Correlation between ankle-brachial index $(A B I)$ oscillometric method results obtained before and after EC (oABI before vs. oABI after, $r=0.49, p<0.001$ ) (A) and Bland-Altman plot between $A B I$ measurements performed before and after EC using oscillometric method (B) 

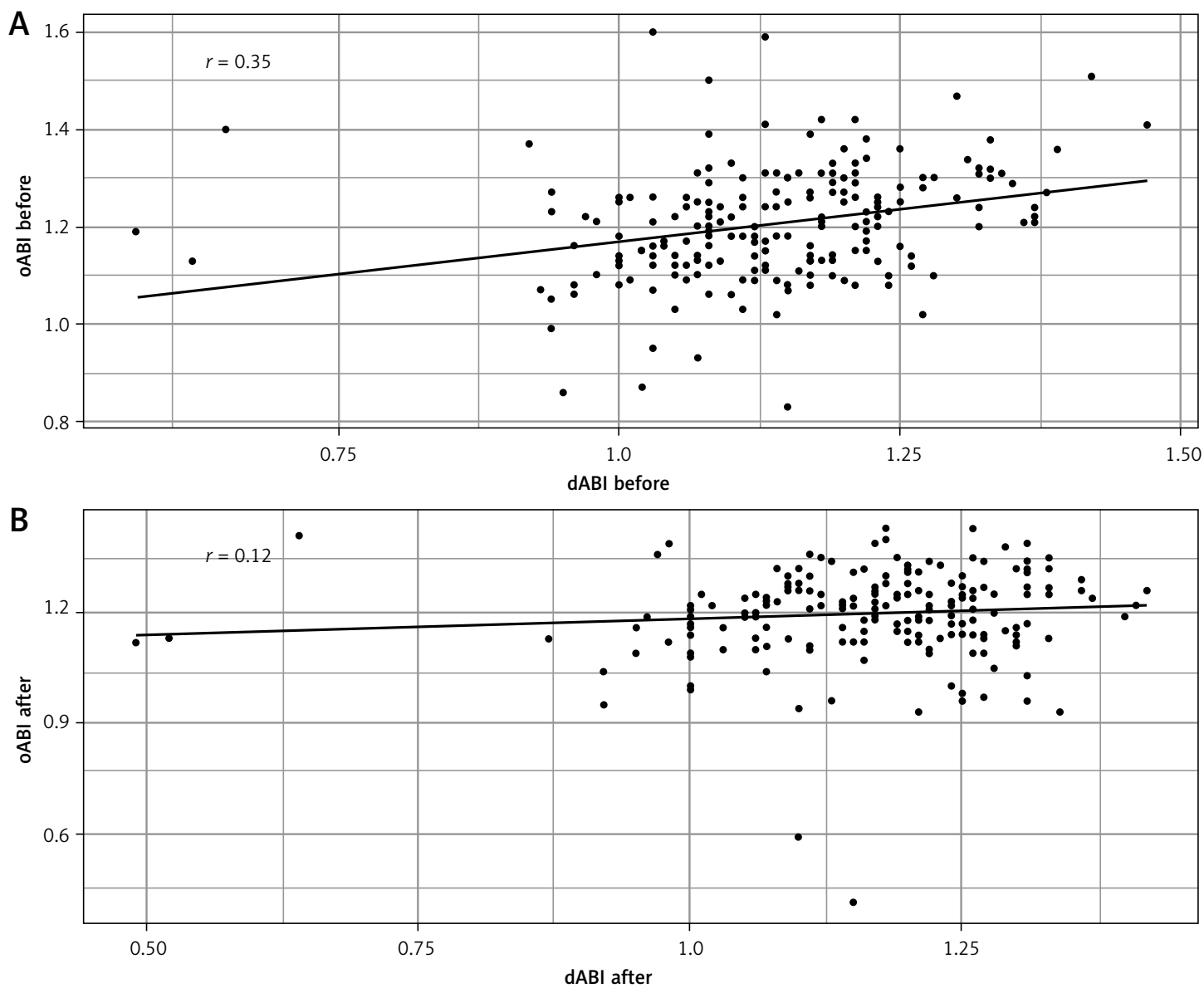

Figure 4. Correlation between ankle-brachial index (ABI) results obtained before EC using Doppler and oscillometric method (dABI before vs. oABI before, $r=0.35, p<0.001 ; A)$ and lack of correlation after $E C$ ( $d A B I$ after vs. oABI after, $r=0.12, p=0.07$; $)$

Arrhythmias, especially atrial fibrillation, are common in the aging population [14]. Unfortunately, atrial fibrillation is often undiagnosed. Atrial fibrillation may substantially influence blood pressure measurements. Previously, we found that the results of $A B I$ measured using the Doppler method during atrial fibrillation corresponded with the results obtained in sinus rhythm [9].

The main finding of the present study is that $A B I$ results obtained using the automated oscillometric method did not differ when measured in sinus rhythm and during atrial fibrillation. The lack of difference between results obtained using the oscillometric method before and after EC does not mean however that the use of the oscillometric method should be preferred for $A B I$ determination in all groups of patients. Previously it was suggested that PAD screening with the oscillometric device may not be suitable in patients with atrial fibrillation [10].

The second important finding of the study is that results of measurements performed using oscillometric and Doppler methods differed from each other. The difference was greater during atrial fibrillation than in sinus rhythm. This is an im- portant observation from the clinical perspective, because it may impact the potential usefulness of automatic oscillometric $A B I$ determination in patients with atrial fibrillation. We observed a weak correlation between the two methods during arrhythmia and no correlation in sinus rhythm, which is in contrast with some other reports [10]. However, the Bland-Altman analysis also showed low agreement of both methods (Figure 5), both during atrial fibrillation and in sinus rhythm, when the Doppler method was considered as the reference. In addition to bias higher than reported by other authors [15], we observed wide limits of agreement, and almost 5\% differences passing the $95 \%$ limit of agreement.

Analyzing the $A B I$ results obtained using the oscillometric method, it is important to realize that the automated method does not measure systolic and diastolic blood pressure directly. The values of systolic and diastolic blood pressure are calculated from the mean blood pressure by the oscillometric device, according to its algorithm. This may cause significant bias, especially during arrhythmia. Because of beat-by-beat changes in pulse pressure and mean pressure, there may be a distortion in 

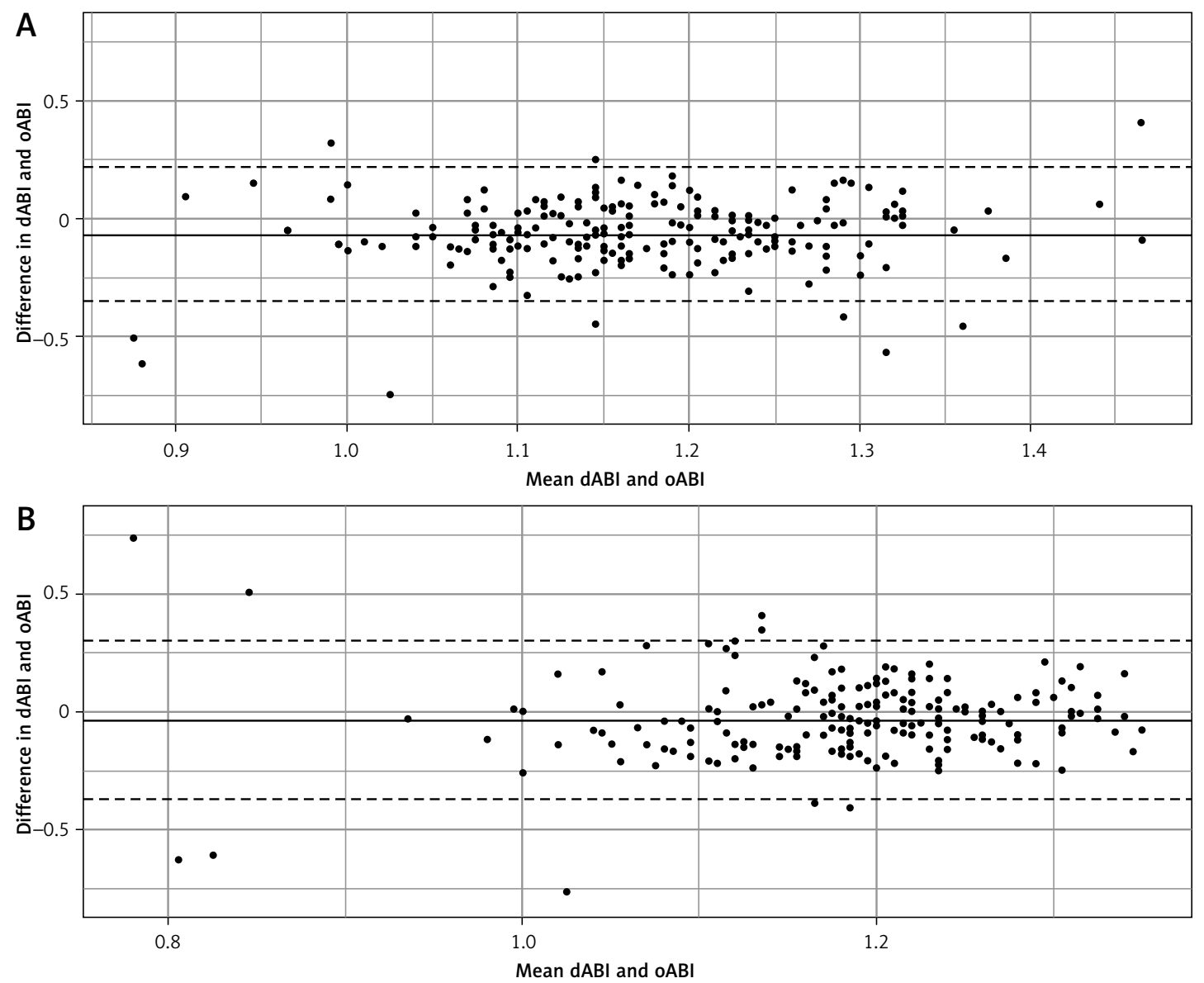

Figure 5. Bland-Altman plot between ankle-brachial index (ABI) measurements performed before EC using Doppler and oscillometric method (A) and after EC (B)

the relation between cuff pressure and oscillometric wave amplitude. Considering that $A B I$ is the ratio between blood pressures, the difference between different measurements may be amplified.

In the medical literature, there is a paucity of information about the $A B I$ measurements in patients with AF. MacDougall et al. showed that the oscillometric method of PAD detection was feasible and operator-independent, even though it did not detect low ABI as efficiently as the Doppler method, especially in subjects with non-palpable peripheral pulses [16]. Hsu et al. found that in patients with $A F$ values of $A B I$ were lower than in subjects with sinus rhythm. After adjustment for heart rate this relation disappeared, which suggests that heart rate can significantly influence the relationship between $A F$ and $A B I$. In the current study, we performed an analysis, dividing studied group into the patients with heart rate (HR) over 100 beats/minute and patients with HR below 100 beats/minute. This approach did not affect differences between results obtained before and after EC and the differences between methods.

In contrast to our study, another work by $\mathrm{Pa}$ gonas et al. [11] found that the biases of systolic and diastolic blood pressure did not significantly differ in the presence or absence of atrial fibrillation, when the Bland-Altman analysis was used for analysis. However, the difference in the results between these two studies may stem from the fact that the authors measured blood pressure, but they did not measure $\mathrm{ABI}$, and that difference impacts the results and any comparisons between these studies. Furthermore, our study analyzed exactly the same patients - before and after EC. That was not the case in the study of Pagonas et al. [11], who analyzed two different populations of patients - with and without atrial fibrillation.

In the current study values of oscillometric measurements were higher than Doppler measurement results. As summarized by Veckber et al., other studies also found higher oscillometric $A B I$ values than the Doppler $A B I$ values. Though from the pathophysiological point of view such a difference should not be present or be opposite, it is probably related to the intrinsic characteristics of each method. Other authors postulated that higher (close to 1) cutoff values for PAD detection should be used using the oscillometric method $[17,18]$.

Automated devices, especially with the AF detection mode, due to easy use are handy tools for 
PAD and possible AF screening. Because of the impact on AF detection automated oscillometric devices may strengthen the appropriate use of the reference Doppler method in case of AF recognition.

The main limitation of the study is that a possible effect of conditions related to the procedure of electrical cardioversion, including effects of anesthesia, cannot be excluded. However, the ABI measurements were performed at the time when propofol should not have direct hemodynamic effects. Furthermore, we designed this research to study in detail the impact of AF on ABI. Therefore, we excluded the hemodynamic effects of the medications that are often prescribed after EC, especially the antiarrhythmic and the heart rate slowing drugs.

The current study identified only a few patients with PAD according to the $A B I$ results $<0.9$, detected by both investigated methods. The oscillometric method is considered as relatively observer-independent, and this study included consecutive patients admitted for EC without prescreening. If the threshold of $A B I<1.0$ to diagnose PAD was used, as suggested by other authors evaluating the oscillometric method $[17,18]$, number of PAD detected would be considerably larger. It worth noting that using the threshold of $A B I<1.0$, numbers of PAD cases detected in the study would be identical using both methods.

In conclusion, postulate that the automated oscillometric method should not replace the reference Doppler method in patients with atrial fibrillation. However, more research related to the oscillometric measurements is needed in subjects with PAD and AF.

\section{Acknowledgments}

This study was supported by Medical University of Warsaw.

\section{Conflict of interest}

The authors declare no conflict of interest.

\section{References}

1. Gerhard-Herman MD, Gornik HL, Barrett C, et al. 2016 AHA/ACC Guideline on the management of patients with lower extremity peripheral artery disease: executive summary: a report of the American College of Cardiology/American Heart Association Task Force on Clinical Practice Guidelines. J Am Coll Cardiol 2017; 69: 1465-508

2. Aboyans V, Ricco JB, Bartelink MEL, Bjorck M, Brodmann M, Cohnert T, et al. 2017 ESC Guidelines on the diagnosis and treatment of peripheral arterial diseases, in collaboration with the European Society for Vascular Surgery (ESVS): document covering atherosclerotic disease of extracranial carotid and vertebral, mesenteric, renal, upper and lower extremity arteriesEndorsed by: the European Stroke Organization (ESO)The Task Force for the Diagnosis and Treatment of Peripheral Arterial Diseases of the European Society of Cardiology (ESC) and of the European Society for Vascular Surgery (ESVS). Eur Heart J 2018; 39: 763-816.

3. Majer M, Gackowski D, Rozalski R, Siomek-Gorecka A, Olinski R, Budzynski J. Systemic oxidoreductive balance and vascular function in individuals without clinical manifestation of atherosclerosis. Arch Med Sci Atheroscler Dis 2017; 2: e37-45.

4. Fowkes FG, Murray GD, Butcher I, et al. Ankle brachial index combined with Framingham Risk Score to predict cardiovascular events and mortality: a meta-analysis. JAMA 2008; 300: 197-208.

5. Carter SA. Indirect systolic pressures and pulse waves in arterial occlusive diseases of the lower extremities. Circulation 1968; 37: 624-37.

6. Yao ST, Hobbs JT, Irvine WT. Ankle systolic pressure measurements in arterial disease affecting the lower extremities. Br J Surg 1969; 56: 676-9.

7. Aboyans V, Criqui MH, Abraham P, et al. Measurement and interpretation of the ankle-brachial index: a scientific statement from the American Heart Association. Circulation 2012; 126: 2890-909.

8. Sykes D, Dewar R, Mohanaruban K, et al. Measuring blood pressure in the elderly: does atrial fibrillation increase observer variability? BMJ 1990; 300: 162-3.

9. Dabrowski M, Lewandowski J, Abramczyk P, Lon I, Gaciong Z, Sinski M. Atrial fibrillation does not affect ankle-brachial index measured using the Doppler method. Hypertens Res 2018; 41: 60-5.

10. Verberk WJ, Kollias A, Stergiou GS. Automated oscillometric determination of the ankle-brachial index: a systematic review and meta-analysis. Hypertens Res 2012; 35: 883-91.

11. Pagonas N, Schmidt S, Eysel J, et al. Impact of atrial fibrillation on the accuracy of oscillometric blood pressure monitoring. Hypertension 2013; 62: 579-84.

12. Stergiou GS, Kollias A, Karpettas N. Does atrial fibrillation affect the automated oscillometric blood pressure measurement? Hypertension 2013; 62: e37.

13. Baker JD, Dix DE. Variability of Doppler ankle pressures with arterial occlusive disease: an evaluation of ankle index and brachial-ankle pressure gradient. Surgery 1981; 89: 134-7.

14. Zembala M, Filipiak K, Kowalski O, et al. Staged hybrid ablation for persistent and longstanding persistent atrial fibrillation effectively restores sinus rhythm in longterm observation. Arch Med Sci 2017; 13: 109-17.

15. Davies JH, Williams EM. Automated plethysmographic measurement of the ankle-brachial index: a comparison with the Doppler ultrasound method. Hypertens Res 2016; 39: 100-6.

16. MacDougall AM, Tandon V, Wilson MP, Wilson TW. Oscillometric measurement of ankle-brachial index. Can J Cardiol 2008; 24: 49-51.

17. Kollias A, Xilomenos A, Protogerou A, Dimakakos E, Stergiou GS. Automated determination of the ankle-brachial index using an oscillometric blood pressure monitor: validation vs. Doppler measurement and cardiovascular risk factor profile. Hypertens Res 2011; 34: 825-30.

18. Korno M, Eldrup N, Sillesen H. Comparison of ankle-brachial index measured by an automated oscillometric apparatus with that by standard Doppler technique in vascular patients. Eur J Vasc Endovasc Surg 2009; 38: 610-5. 